\title{
Right-Sided Arcus Aorta Syndrome
}

\author{
Sevket Ozkaya ${ }^{\mathrm{a}, \mathrm{e}}$, Adem Dirican ${ }^{\mathrm{b}}$, Koksal Atalay ${ }^{\mathrm{c}}$, Bilal Sengul $^{\mathrm{d}}$, Ferah Ece ${ }^{\mathrm{a}}$
}

\begin{abstract}
Right-sided arcus aorta (RSAA) syndrome was new defined in patients with chronic chough and exertional dyspnea. The new six patients with RSAA syndrome were diagnosed in Samsun Medicalpark Hospital, Department of Pulmonary Medicine between July 2012 and December 2013. The ages of the patients ranged from 4 to 78 years and the male to female ratio was $4 / 2$. Three patients were symptomatic due to external compression of the trachea due to right aortic arch. The most common symptoms were exertional dyspnea and chronic cough. In conclusion, in this study, the RSAA syndrome is supported with new patients. We think that it is more common than estimated and all patients with exertional dyspnea and chronic cough should be evaluated for the RSAA syndrome. This syndrome should be considered and can be determined in all age groups.
\end{abstract}

Keywords: Right-sided arcus aorta syndrome; Cough; Dyspnea

Manuscript accepted for publication October 13, 2014

aDepartment of Pulmonary Medicine, Faculty of Medicine, Bahcesehir University, Istanbul, Turkey

${ }^{b}$ Department of Pulmonary Medicine, Samsun Medicalpark Hospital, Samsun, Turkey

'Department of Radiology, Samsun Medicalpark Hospital, Samsun, Turkey

${ }^{\mathrm{d}}$ Department of Pulmonary Medicine, Samsun Chest Diseases and Thoracic Surgery Hospital, Samsun, Turkey

${ }^{\mathrm{e} C o r r e s p o n d i n g ~ A u t h o r: ~ S e v k e t ~ O z k a y a, ~ D e p a r t m e n t ~ o f ~ P u l m o n a r y ~ M e d i c i n e, ~}$ Faculty of Medicine, Bahcesehir University, Istanbul, Turkey.

Email: ozkayasevket@yahoo.com

doi: http://dx.doi.org/10.14740/jmc1965w

\section{Introduction}

Right-sided arcus aorta (RSAA) syndrome was defined in patients with chronic chough and exertional dyspnea [1]. Also, we suggested that the RSAA syndrome should be included in the differential diagnosis of asthma [2-4]. We want to present new patients with RSAA syndrome.

\section{Case Reports}

The new patients with RSAA syndrome were diagnosed in Samsun Medicalpark Hospital, Department of Pulmonary Medicine between July 2012 and December 2013. The characteristics of patients including age, gender, symptoms, and radiological and spirometric findings were retrospectively evaluated and presented. Written consents of our patients and the approval of the institution were obtained to carry out our study.

The six new patients with RSAA syndrome were diagnosed and the characteristics of the patients were presented in Table 1 . The ages of the patients ranged from 4 to 78 years and the male to female ratio was $4 / 2$. Three patients $(50 \%)$ were symptomatic due to external compression of the trachea due to right aortic arch (Figs. 1-5). The most common symptoms were exertional dyspnea and chronic cough. The symptoms of the other three patients were caused from lung cancer, COPD and hypertension with chronic renal failure.

The spirometry flow-volume curves throughout exhalation and inhalation showed intrathoracic tracheal obstruction in two patients (Fig. 4). Other spirometric findings were restrictive in one patient and obstructive in one patient. Spirometry was normal in one patient. The diagnosis of RSAA was confirmed by thoracic CT and/or MRI in all patients. Thoracic MRI also revealed marked narrowing of the tracheal air column due to external compression by the RSAA in three patients (Figs. 1, 2 and 4).

\section{Discussion}

We previously reported the RSAA together with dyspnoea and cough during exercise is a new clinical entity that should be called RSAA syndrome [1]. Although the incidence of RSAA 


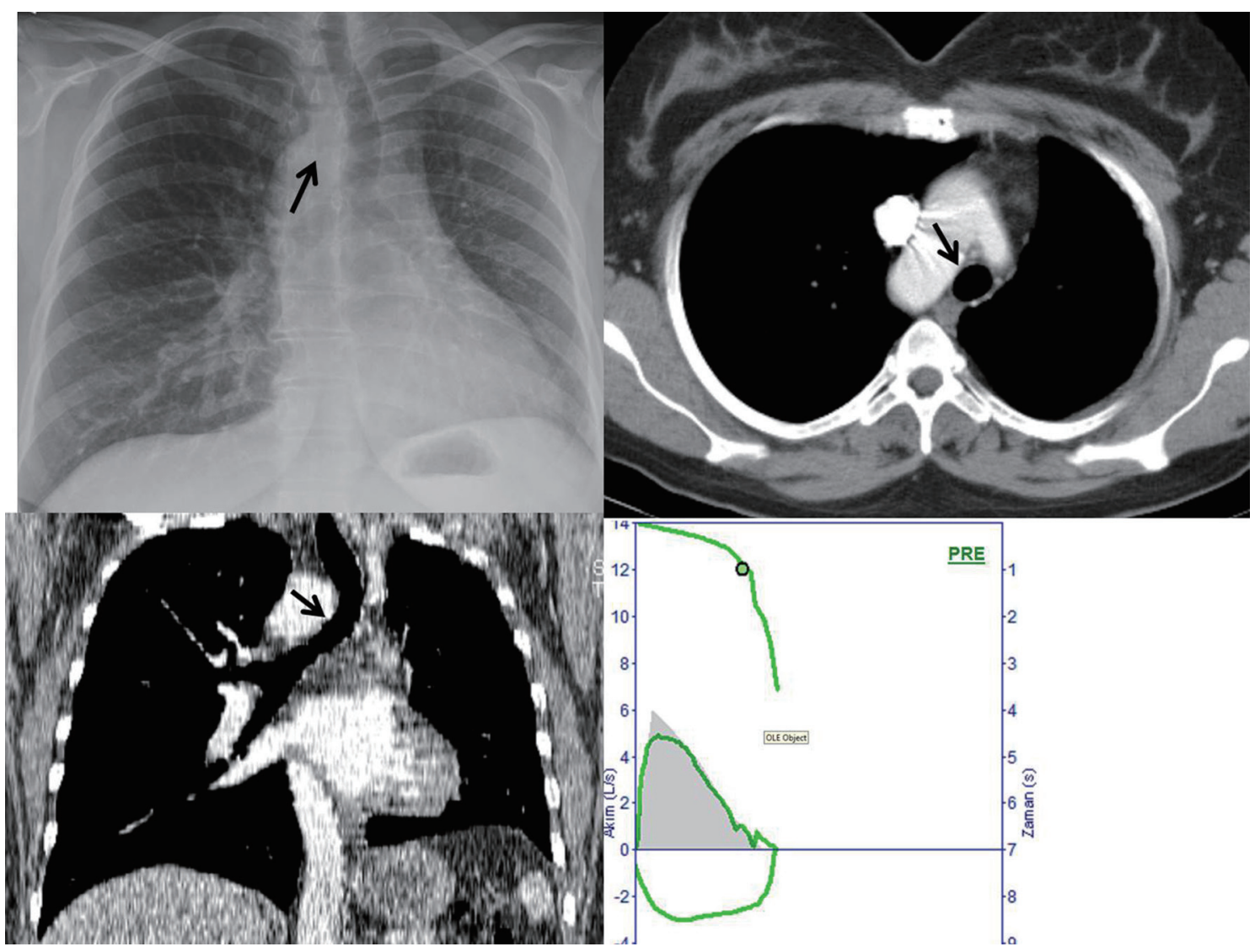

Figure 1. Patient no. 2. A 47-year-old female patient was admitted to hospital with complaints of chronic cough and dyspnea. Thorax radiography and CT images revealed right-sided arcus aorta with tracheal compression. Spirometry showed a normal flow-volume curve.

is variying, it is much more than estimated. The incidence of RSAA was reported to be $0.16 \%$ [3]. Approximately $50 \%$ of patients were symptyomatic due to RSAA. In the present study, the ages of patients ranged from 4 to 78 years and two of them were female. The most common reported symptoms were dyspnea and chronic cough especially with exercise. In this case series, $50 \%$ of were symptomatic due to external com- pression of the trachea due to right aortic arch. The most common symptoms were exertional dyspnea and chronic cough. The symptoms of other three patients were caused from lung cancer, COPD and hypertension with chronic renal failure. Spirometry can be helpful in the diagnosis of suspected tracheal compression in symptomatic patients. The plateau of expirium loop of flow-volume curves on spirometry is a message

Table 1. The Characteristics of Patients

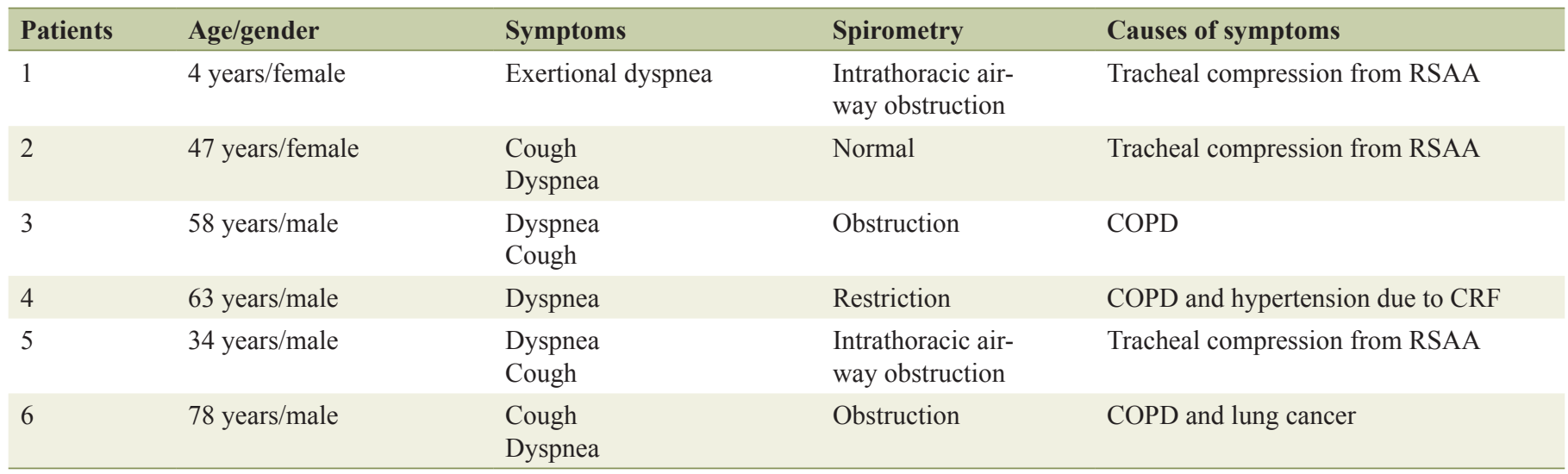




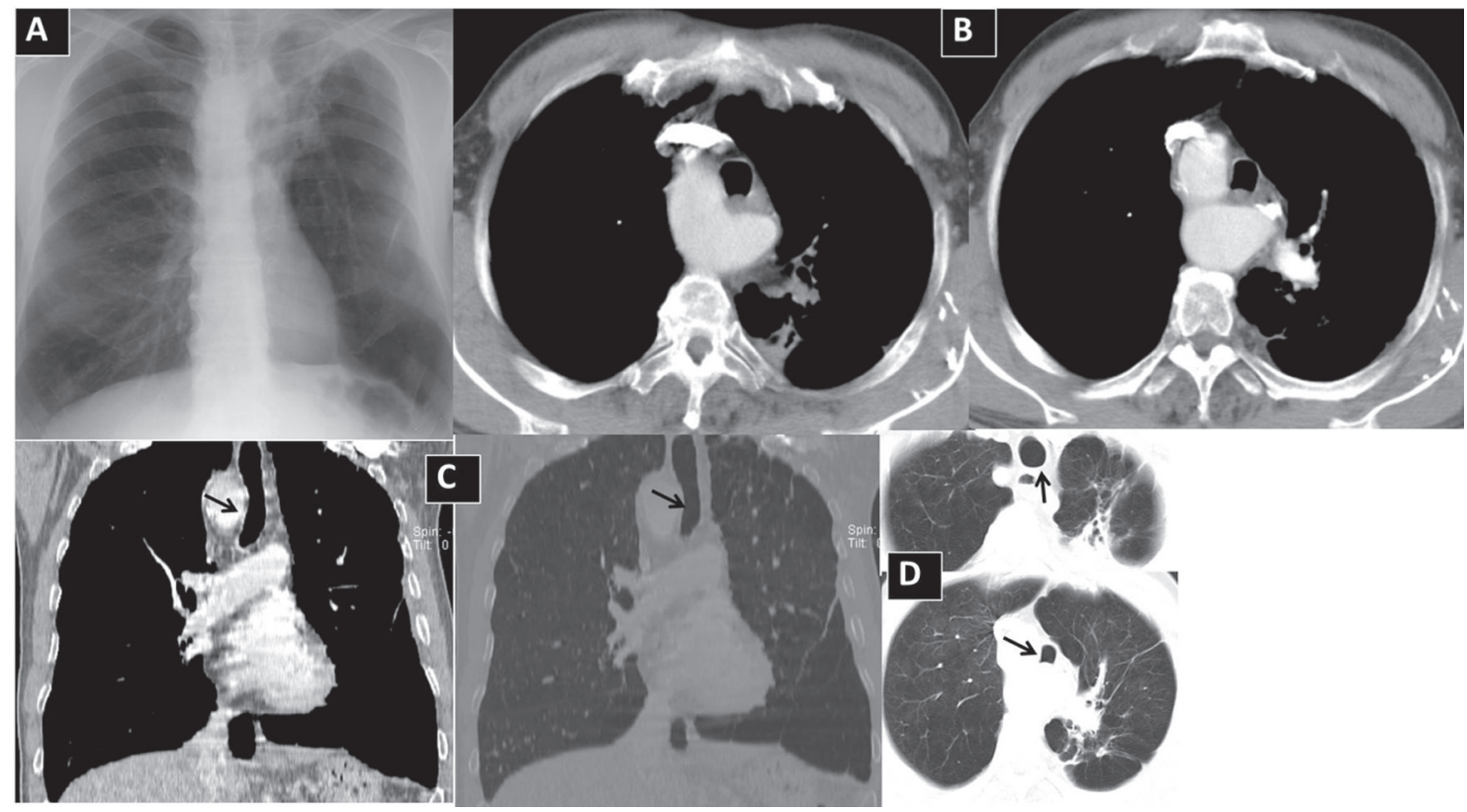

Figure 2. Patient no. 3. A 58-year-old male patient was admitted to hospital with complaints of cough and dyspnea. Thorax radiography and CT images showed right-sided arcus aorta with tracheal compression (arrows on Figure $1 \mathrm{~A}-\mathrm{C}$ ). The diameter of trachea is seen normal above the aortic arch. But the diameter of trachea is seen significantly narrowed at the same level of arcus aorta due to compression (arrows on Figure 1D).

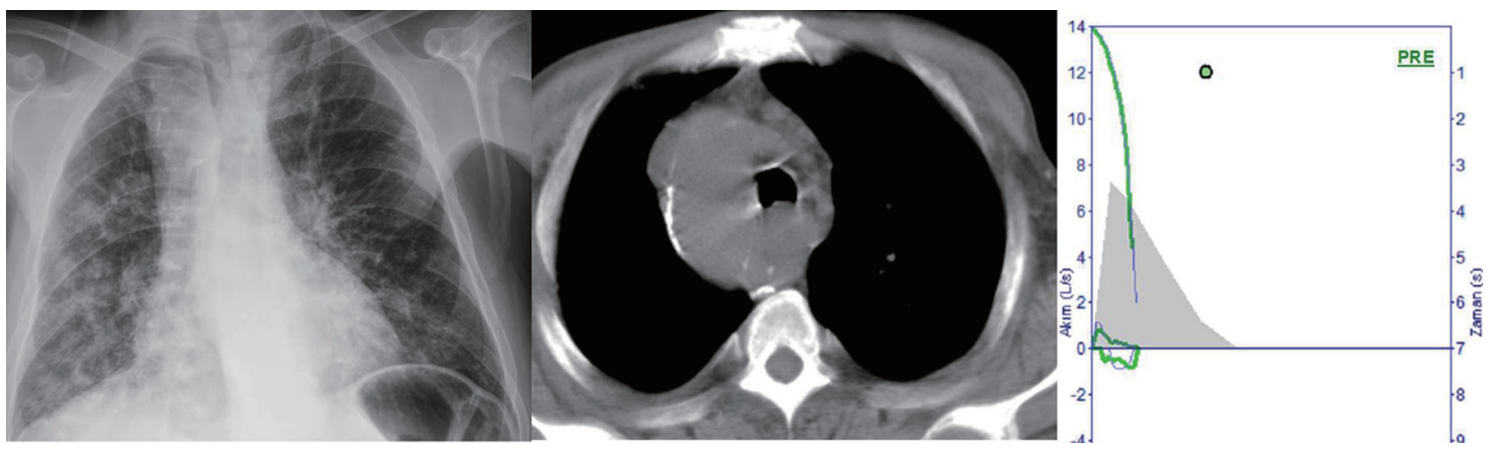

Figure 3. Patient no. 4. A 63-year-old man was admitted to hospital with dyspnea. Chest radiography showed that the infiltration and shadow of arcus aorta is seen on the right side of mediastinum. The right-sided arcus aorta was confirmed with thorax CT. Pulmonary function test revealed the obstruction.

for diagnosis to clinicians. The spirometry flow-volume curves throughout exhalation and inhalation showed intrathoracic tracheal obstruction in two of patients with RSAA syndrome. Spirometry can be normal in symptomatic patients with RSAA syndrome, as seen in one of our patients. The most common radiologic finding, the shadow of arcus aorta was not seen on the left side of the mediastinum with chest radiography. One patient was with RSAA syndrome. Thoracic CT and MRI are the best methods for diagnosis of RSAA. MRI should be the preferred diagnostic test. MRI is a non-invasive diagnostic tool and can clearly show the relationship between intrathoracic vascular structures and the trachea. As we emphasized in all of our previous studies, RSAA syndrome should be included in the differential diagnosis of asthma, and all patients with intractable and exertional dyspnoea should be evaluated for RSAA anomaly [1-4].

In conclusion, in this study, the RSAA syndrome is supported with new patients. We think that it is more common than estimated and all patients with exertional dyspnea and chronic cough should be evaluated for the RSAA syndrome. This syndrome should be considered and can be determined in all age groups.

\section{Conflicts of Interest}

The authors report no conflicts of interest. 

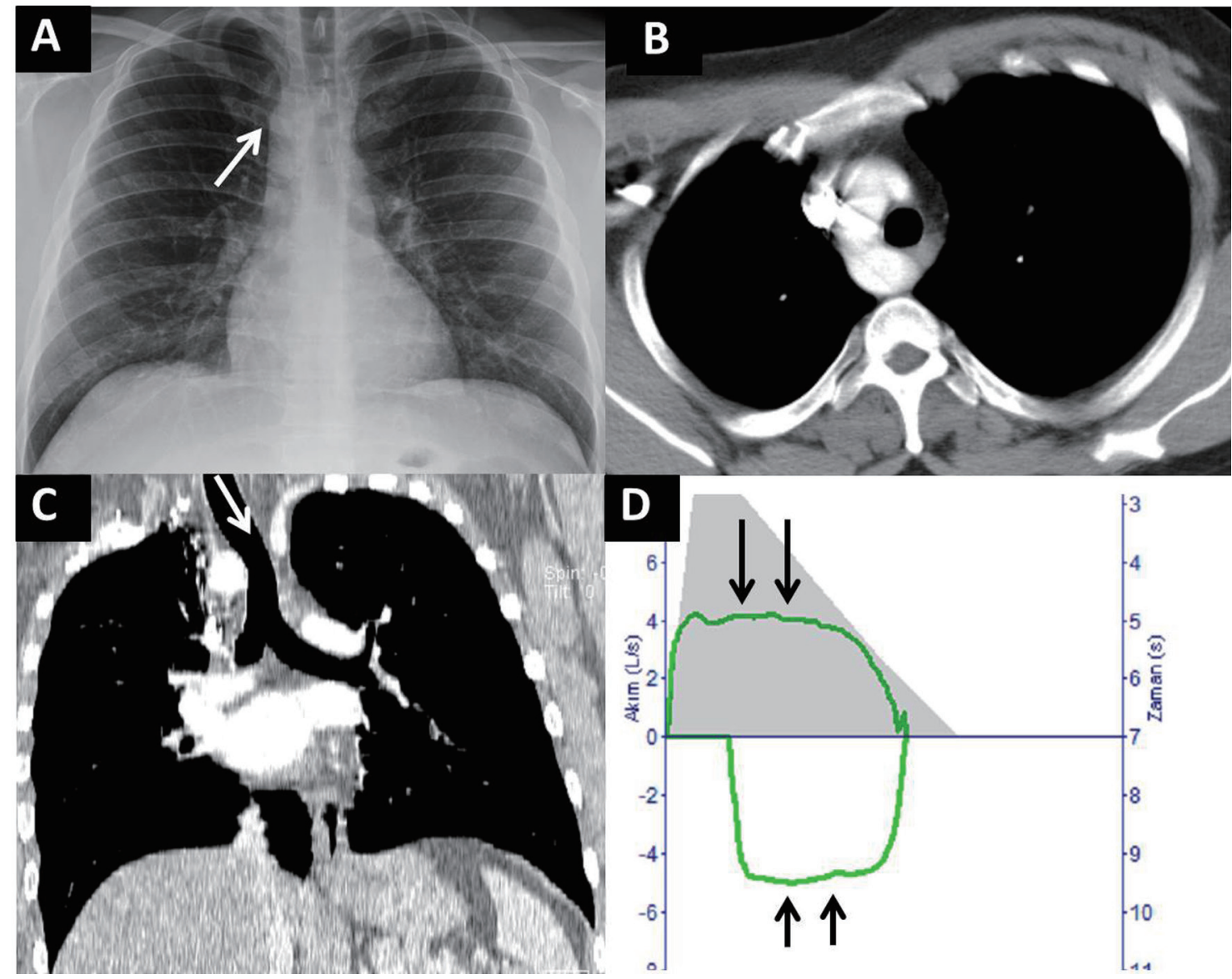

Figure 4. Patient no. 5. A 24-year-old man suffered from dyspnea and cough, especially with exercise. Thorax radiography and CT images showed right-sided arcus aorta with tracheal compression. Marked compression of the trachea is seen on thoracic CT images (arrows on Figure 4A-C). Pulmonary function test showed the plateau on flow-volume curves throughout exhalation and inhalation (arrows on Figure 4C).

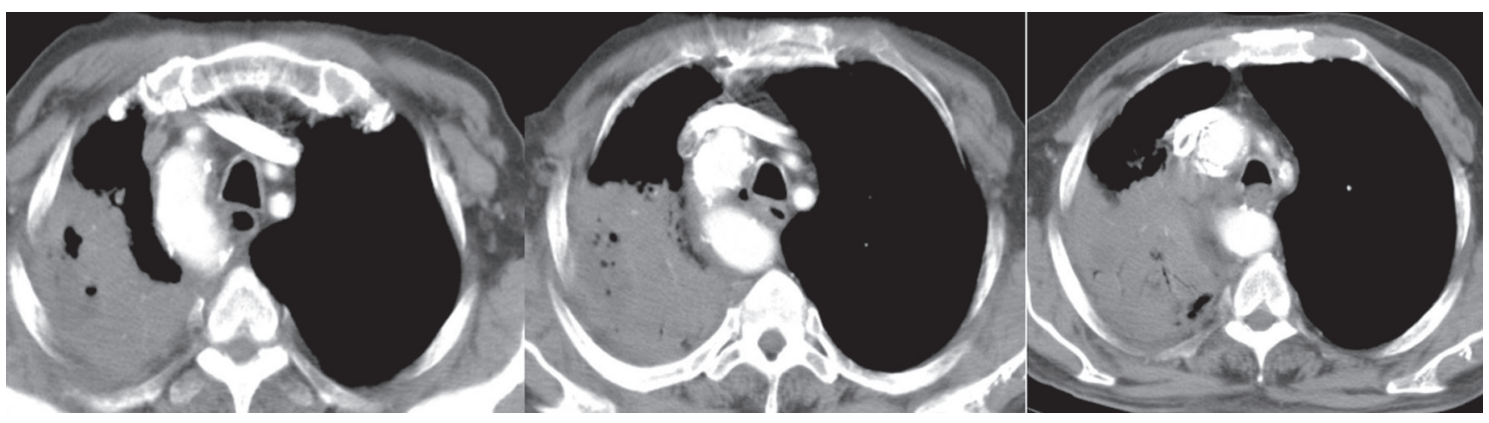

Figure 5. Patient no. 6. A 78-year-old man presented with lung cancer and COPD. Thorax radiography and CT images showed right-sided arcus aorta and pulmonary mass.

\section{References}

1. Ozkaya S, Dirican A, Tuna T, Ece F. A new syndrome: right-sided arcus aorta syndrome. BMJ Case Rep. 2013;2013.

2. Ozkaya S, Sengul B, Hamsici S. A Rare Cause of Intrathoracic Upper Airway Obstruction. Tur Toraks Der.
2009;10:37-38.

3. Ozkaya S, Sengul B, Hamsici S, Findik S. An unusual cause of dyspnea. J Asthma. 2010;47(8):946-948.

4. Ozkaya S, Sengul B, Hamsici S, Findik S, Sahin U, Gumus A, Cinarka H. Right sided arcus aorta as a cause of dyspnea and chronic cough. Multidiscip Respir Med. 2012;7(1):37. 